ESAIM: COCV

Vol. 13, No 1, 2007, pp. 107-119

DOI: $10.1051 /$ cocv:2007005
ESAIM: Control, Optimisation and Calculus of Variations

www.edpsciences.org/cocv

\title{
HOMOGENIZATION OF HAMILTON-JACOBI EQUATIONS IN CARNOT GROUPS
}

\author{
Bianca Stroffolini ${ }^{1}$
}

\begin{abstract}
We study an homogenization problem for Hamilton-Jacobi equations in the geometry of Carnot Groups. The tiling and the corresponding notion of periodicity are compatible with the dilatations of the Group and use the Lie bracket generating property.
\end{abstract}

Mathematics Subject Classification. 35B27, 35H05.

Received March 16, 2005.

\section{BACKGROUND AND MOTIVATIONS}

Consider a Hamilton-Jacobi equation:

$$
u+H(\xi, \nabla u)=0 \quad \text { in } \quad \mathbb{R}^{N}
$$

where the Hamiltonian $H(\xi, p): \mathbb{R}^{N} \times \mathbb{R}^{N \times N} \rightarrow \mathbb{R}$ is not coercive in $p$. The lack of coerciveness of the Hamiltonian can be overcome by changing the underlying geometry with a suitable family of vector fields. More precisely, we are able to consider the case when $H(\xi, p)=H(\xi, \sigma(\xi) q)$, where $\sigma(\xi)$ is a $m \times N$ matrix, $m<N$, $H$ is coercive in $q$. Here the rows of the matrix $\sigma(\xi)$ will be considered as coefficients of vector fields satisfying Hörmander condition and that generate a Carnot Group, therefore $\sigma(\xi) \nabla u$ will be the horizontal gradient in a Carnot Group, denoted by $D_{h} u$, see Section 2 .

We shall consider homogenizations problems for Hamilton-Jacobi equations of the form:

$$
u^{\varepsilon}(\xi)+H\left(\xi, \frac{\xi}{\varepsilon}, D_{h} u^{\varepsilon}(\xi)\right)=0
$$

in a Carnot Group $G$, where the Hamiltonian $H$ is $G$-periodic in the second variable and $\frac{\xi}{\varepsilon}$ will be interpreted in the geometry of the group.

Let us revise first some key results in the Euclidean setting, for $\mathbb{Z}^{N}$-periodic Hamiltonians. The pioneering paper on homogenization of Hamilton-Jacobi equations is due to P.L. Lions, Papanicolau and Varadhan. They proved that the asymptotic behaviour, as $\varepsilon$ tends to zero, of the solutions $u^{\varepsilon}$ is governed by the equation:

$$
u(\xi)+\bar{H}(\xi, D u(\xi))=0 .
$$

Keywords and phrases. Homogenization, Carnot Groups, Hamilton-Jacobi.

1 Dipartimento di Matematica e Applicazioni Università degli studi di Napoli Federico II Complesso Monte S. Angelo Edificio "T" via Cintia, 80126 Napoli Italy; bstroffo@unina.it

(c) EDP Sciences, SMAI 2007 
The effective Hamiltonian $\bar{H}$ is obtained by solving a cell problem:

$$
H(x, \xi, p+D v(\xi))=\lambda
$$

for every $(x, p)$ fixed and putting $\bar{H}(x, p)=\lambda$.

Evans $[8,9]$ developed the perturbed test functions method for homogenization problems in the framework of the theory of viscosity solutions, see also [4]. The combined use of comparison results and perturbed test functions allow to establish, in a simple way, uniform estimates in the sup norm for the solutions $u^{\varepsilon}$ to the solution of the homogenized equation.

The question of estimating the rate of the uniform convergence of the solutions $u^{\varepsilon}$ to the solution of the homogenized equation has been tackled up by Capuzzo Dolcetta and Ishii [7].

For recent developments in the framework of stochastic control and second-order Hamilton-Jacobi-Bellmann equations, see the papers $[1,2]$.

Birindelli and Wigniolle have studied the problem in the Heisenberg Group, [6], giving also an estimate of the rate of convergence.

The aim of this paper is the homogenization in general Carnot Group of step $r$, with a strong interplay with their geometry. We reinterpret the results obtained "by hand" by Birindelli and Wigniolle, in the geometry of the group, using the Lie bracket generating property, and a geometric construction of the tiling, valid in any Carnot Group.

In the Heisenberg Group case, the authors were able to give estimates of the rate of convergence, using the homogeneity of test functions of degree two, and, therefore, uniform estimates of the second derivatives; for step $r$ groups, $r>2$, we will obtain uniform convergence of $u^{\varepsilon}$ towards the solution of the homogenized equation.

Notice that viscosity solutions are Lipschitz continuous with respect to the group distance, but only Hölder continuous with respect to the Euclidean one. Viscosity solutions for Hamilton-Jacobi equations in the Heisenberg Group were considered in [16].

\section{Carnot Groups}

We will consider $\left(\mathbb{R}^{N}, \cdot\right)$ as a Carnot Group with a Group operation · and a family of dilations, compatible with the Lie structure.

Let us briefly recall some basic facts about Carnot Groups $G$. A Carnot Group $G$ of step $r \geq 1$, is a simply connected nilpotent Lie Group whose Lie algebra $\mathfrak{g}$ is stratified. This means that $\mathfrak{g}$ admits a decomposition as a vector space sum

$$
\mathfrak{g}=\mathfrak{g}_{1} \oplus \mathfrak{g}_{2} \oplus \cdots \oplus \mathfrak{g}_{r}
$$

such that

$$
\left[\mathfrak{g}_{1}, \mathfrak{g}_{j}\right]=\mathfrak{g}_{j+1}
$$

for $j=1 \cdots r$ with $\mathfrak{g}_{k}=\{0\}$ for $k>r$. Note that $\mathfrak{g}$ is generated as a Lie algebra by $\mathfrak{g}_{1}$.

Let $m_{j}=\operatorname{dim} \mathfrak{g}_{j}$ and chose a basis of $\mathfrak{g}_{j}$ formed by left-invariant vector fields $X_{i, j}, i=1, \cdots, m_{j}$. The dimension of $G$ as a manifold is $N=m_{1}+\cdots m_{r}$. The horizontal tangent space at a point $\xi \in G$ is the $m_{1^{-}}$ dimensional subspace that is linearly spanned by $X_{1,1}(\xi), \cdots X_{m_{1}, 1}(\xi)$. From now on, we will indicate simply by $m$ the dimension of $G_{1}$ and by by $X_{1}, \cdots X_{m}$ a frame of vector fields that span the first layer $G_{1}$. The exponential coordinates are given by the diffeomorphism $F: \mathbb{R}^{N} \rightarrow G$ defined by:

$$
F(x)=\exp \left(\sum_{j=1}^{r} \sum_{i=1}^{m_{j}} x_{i, j} X_{i, j}\right) .
$$

Denoting by - the group operation in $G$, the mapping $(\xi, \eta) \rightarrow \xi \cdot \eta$ has polynomial entries, when written in exponential coordinates. Viceversa, given a point $\xi \in G$, we denote by $x_{s}$ the vector of coordinates $\left(x_{1, s}, \cdots, x_{m_{s}, s}\right)$ 
for every $s=1, \cdots, r$. The following formula holds:

$$
X_{\alpha}=\frac{\partial}{\partial x_{1, \alpha}}+\sum_{s=2}^{r} \sum_{\beta=1}^{m_{s}} b_{s, \alpha}^{\beta}\left(x_{1}(\xi), \cdots, x_{s-1}(g)\right) \frac{\partial}{\partial x_{s, \beta}}, \cdots \alpha=1, \cdots, m
$$

where each $b_{s, \alpha}^{\beta}$ is a polynomial of weighted degree $s-1$. By weighted degree we mean that the layer $\mathfrak{g}_{s}$ in the stratification of $\mathfrak{g}$ has the degree $\mathrm{s}$. Correspondingly, each homogeneous monomial $x_{1}^{\alpha_{1}}, \cdots, x_{r}^{\alpha_{r}}$, with multi-indices $\alpha_{s}=\left(\alpha_{1, s}, \cdots, \alpha_{m_{s}, s}\right), s=1, \cdots, r$ is said to have weighted degree $p$ if

$$
\sum_{s=1}^{r} s\left(\sum_{\beta=1}^{m_{s}} \alpha_{\beta, s}\right)=p
$$

There is a family of dilations that is compatible with the group operation:

$$
\delta_{\lambda}\left(x_{1}, \cdots, x_{N}\right)=\left(\lambda x_{1,1}, \cdots, \lambda x_{1, m}, \lambda^{2} x_{2,1}, \cdots, \lambda^{2} x_{2, m_{2}}, \cdots, \lambda^{r} x_{r, m_{r}}\right) .
$$

With the above notations the horizontal subspace can be identified with the left translation by $\xi$ of $G_{1}$, that is we have

$$
\xi \cdot G_{1}=\text { Linear-span }\left\{X_{1}(\xi), \cdots, X_{m}(\xi)\right\} .
$$

A horizontal curve $\gamma(t)$ is a piece-wise smooth curve whose tangent vector $\gamma^{\prime}(t)$ is in the the horizontal tangent space $(\gamma(t)) \cdot G_{1}$ whenever it exists. Given two points $\xi$ and $\eta$ we consider the set of all possible horizontal curves joining these points:

$$
\Gamma(\xi, \eta)=\{\gamma \text { horizontal curve }: \gamma(0)=\xi, \gamma(1)=\eta\}
$$

This set is never empty by Chow's accessibility theorem (see for example [5]). For convenience, fix an ambient Riemannian metric in $\mathfrak{g}$ so that $\mathfrak{X}=\left\{X_{i, j}\right\}$ is an orthonormal frame and the

Riemannian volume element $=$ Haar measure of $G=$ Lebesgue measure in $\mathbb{R}^{N}$.

The Carnot-Carathéodory distance is then defined as the infimum of the length of horizontal curves of the set $\Gamma$ :

$$
d_{C C}(\xi, \eta)=\inf _{\Gamma(\xi, \eta)} \int_{0}^{1}\left|\gamma^{\prime}(t)\right| \mathrm{d} t
$$

It depends only of the restriction of the ambient Riemannian metric to the horizontal distribution generated by the horizontal tangent spaces.

The Carnot-Carathéodory ball of radius $R$ centered at a point $\xi$ is given by

$$
B(\xi, R)=\left\{\eta \in G: d_{C C}(\xi, \eta)<r\right\}
$$

The Carnot-Carathéodory gauge is given by

$$
|\xi|_{C C}=d(0, \xi)
$$

An important cone-like property of this gauge is that it solves the eikonal equation in the almost everywhere sense. This was proven by Monti and Serra-Cassano [19] in general Carnot Groups.

Theorem 1. Consider the horizontal gradient of $|\xi|_{C C}$ given by

$$
X\left(|\xi|_{C C}\right)=\left(X_{1}\left(|\xi|_{C C}\right), \cdots, X_{m}\left(|\xi|_{C C}\right)\right) .
$$

Then, for a.e. $\xi \in \mathbb{R}^{N}$, we have

$$
\left|X\left(|\xi|_{C C}\right)\right|=1
$$


A smooth gauge in $G$ is defined:

$$
|\xi|_{G}=\left(\sum_{j=1}^{r}\left(\sum_{i=1}^{m_{j}}\left|x_{i, j}\right|^{2}\right)^{\frac{r !}{j}}\right)^{\frac{1}{2 r !}} .
$$

Theorem 2 [5].

$$
\begin{gathered}
|\xi|_{C C} \sim|\xi|_{G} \sim \sum_{j=1}^{r} \sum_{i=1}^{m_{j}}\left|x_{i, j}\right|^{\frac{1}{j}} \\
\operatorname{vol}(B(0, R)) \sim r^{Q}
\end{gathered}
$$

where $Q=\sum_{j=1}^{r} j m_{j}$ is the homogeneous dimension of $G$.

From the point of view of the viscosity theory, we do know that the smooth gauge is a viscosity subsolution of the eikonal equation, see Section 3.

For simplicity, we will make the following assumptions on $G$ : denote by $X_{1}, \cdots, X_{m}$ a basis for the first layer and suppose that the following identities hold:

$$
\left[X_{1}, X_{m}\right]=X_{m+1}, \cdots,\left[X_{1}, X_{m+r-1}\right]=X_{m+r}, \quad m+r=N .
$$

Following $[5,10]$, the group operation in $G$ has polynomial entries:

$$
\begin{gathered}
\xi \cdot \eta=\left(x_{1,1}+y_{1,1}, \cdots, x_{1, m}+y_{1, m}, x_{m+1}+y_{m+1}+Q_{2}\left(x_{1, j}, y_{1, j}\right),\right. \\
\left.x_{m+2}+y_{m+2}+Q_{3}\left(x_{1, j}, y_{1, j}, x_{m+1}, y_{m+1}\right), \cdots, x_{m+r}+y_{m+r}+Q_{r}\left(x_{1, j}, y_{1, j}, \cdots, x_{m+r-1}, y_{m+r-1}\right)\right)
\end{gathered}
$$

where each $Q_{s}, s=1, \cdots, r$ is an homogeneous polynomial of degree $s$ in the variables indicated.

\subsection{Examples}

\subsubsection{Heisenberg Group}

Such group is the simplest prototype of a Carnot Group of step two. It can be identified with the Euclidean space $\mathbb{C}^{n} \times \mathbb{R} \equiv \mathbb{R}^{2 n+1}$ endowed with the non-abelian group law:

$$
(x, y, t) \cdot\left(x^{\prime}, y^{\prime}, t^{\prime}\right)=\left(x+x^{\prime}, y+y^{\prime}, t+t^{\prime}+\frac{1}{2}\left\langle x, y^{\prime}\right\rangle-\left\langle x^{\prime}, y\right\rangle\right) .
$$

The Heisenberg algebra is splitted in $V_{1} \oplus V_{2}$, where $V_{1}=\mathbb{R}^{2 n} \times\{0\}$ and $V_{2}=\{0\} \times \mathbb{R}$. It is generated by the vector fields:

$$
\begin{aligned}
& X_{j}=\frac{\partial}{\partial x_{j}}+\frac{1}{2} y_{j} \frac{\partial}{\partial t} \\
& Y_{j}=\frac{\partial}{\partial y_{j}}-\frac{1}{2} x_{j} \frac{\partial}{\partial t}
\end{aligned}
$$

The only nontrivial commutator is

$$
\left[X_{j}, Y_{j}\right]=\frac{\partial}{\partial t}
$$

The homogeneous dimension is 4 . 


\subsubsection{Engel Group}

The Engel Group $\mathcal{E}$ can be identified with $\mathbb{R}^{4}$ and the Lie algebra is decomposed in:

$$
\mathfrak{E}=G_{1} \oplus G_{2} \oplus G_{3}
$$

The horizontal algebra is spanned by $X_{1}, X_{2}$ and the nontrivial commutators are:

$$
\left[X_{1}, X_{2}\right]=X_{3} \quad\left[X_{1}, X_{3}\right]=\left[X_{1},\left[X_{1}, X_{2}\right]\right]=X_{4}
$$

This is a group of step 3 .

The group law is:

$$
(x, y, t, s) \cdot\left(x^{\prime}, y^{\prime}, t^{\prime}, s^{\prime}\right)=\left(x+x^{\prime}, y+y^{\prime}, t+t^{\prime}+Q_{2}, s+s^{\prime}+Q_{3}\right)
$$

where

$$
\begin{gathered}
Q_{2}=\frac{1}{2}\left(x y^{\prime}-y x^{\prime}\right) \\
Q_{3}=\frac{1}{2}\left(x t^{\prime}-t x^{\prime}\right)+\frac{1}{12}\left(x^{2} y^{\prime}-x x^{\prime}\left(y+y^{\prime}\right)+y x^{2}\right) .
\end{gathered}
$$

The homogeneous dimension is 7. For more examples of Carnot Groups, see the recent monograph [17].

We recall the following property, that in the sequel will be referred as the Lie bracket generating property. Following the notations of [20] and [19], we define the following quantities. Let $S_{1}, \cdots, S_{\ell}$ be fields belonging to the family $X_{1}, \cdots, X_{m}$ and let $a \in R$ :

and, by induction:

$$
\begin{gathered}
C_{1}\left(a ; S_{1}\right)=\exp \left(a S_{1}\right) \\
C_{2}\left(a ; S_{1}, S_{2}\right)=\exp \left(-a S_{2}\right) \exp \left(-a S_{1}\right) \exp \left(a S_{2}\right) \exp \left(a S_{1}\right)
\end{gathered}
$$

$$
C_{\ell}\left(a ; S_{1}, \cdots, S_{\ell}\right)=C_{\ell-1}\left(a ; S_{2}, \cdots, S_{\ell}\right)^{-1} \exp \left(-a S_{1}\right) C_{\ell-1}\left(a ; S_{2}, \cdots, S_{\ell}\right) \exp \left(a S_{1}\right) .
$$

By the Campbell-Hausdorff formula and the Jacobi identity they get the following equality:

$$
C_{2}\left(a ; S_{1}, S_{2}\right)=\exp \left(a^{2}\left[S_{1}, S_{2}\right]+\sum_{d(I)>2} c_{I} a^{d(I)} S_{[I]}\right) .
$$

Iterating:

$$
C_{\ell}\left(a ; S_{1}, S_{2}, \cdots, S_{\ell}\right)=\exp \left(a^{\ell} S_{[1, \cdots, \ell]}+\sum_{d(I)>\ell} c_{I} a^{d(I)} S_{[I]}\right) .
$$

Here $d(I)$ represents the length of the multiindex $I$, so the iteration will end after $r$ steps, where $r$ is the step of the group.

\section{HAMILTON-JACOBI EQUATION}

In order to define viscosity solutions we must first identify the first order jets adapted to our framework. Motivated by the Taylor expansion $[5,15]$ consider a differentiable function $u: G \mapsto \mathbb{R}$ at the point $\xi_{0}$. We have

$$
u(\xi)=u\left(\xi_{0}\right)+\left\langle D_{\mathrm{h}} u\left(\xi_{0}\right), \overline{\xi_{0}^{-1} \cdot \xi}\right\rangle+o\left(\left|\xi_{0}^{-1} \cdot \xi\right|_{G}\right),
$$

where $D_{\mathrm{h}} u=X_{1} u X_{1}+\cdots+X_{m} u X_{m}$ is the horizontal gradient of $u$ and $\bar{\xi}$ is the horizontal projection. 
Definition 1. A function $u$ : $G \mapsto \mathbb{R}$ is of class $C^{1}$ if the horizontal derivatives $X_{1} u, \cdots, X_{m} u$ are continuous.

If a function $u$ is not necessarily smooth but merely upper semicontinuous, the collection of vectors $p \in \mathbb{R}^{m}$ such that

$$
u(\xi) \leq u\left(\xi_{0}\right)+\left\langle p, \overline{\xi_{0}^{-1} \cdot \xi}\right\rangle+o\left(\left|\xi_{0}^{-1} \cdot \xi\right|_{G}\right)
$$

is denoted by $J_{u}^{1,+}\left(\xi_{0}\right)$ and called the first order superjet of $u$ at the point $\xi_{0}$. Analogously we define $J_{v}^{1,-}\left(\xi_{0}\right)$, the first order subjet of a lower semicontinuous function $v$ at $\xi_{0}$ as the set of vectors $q \in \mathbb{R}^{m}$ such that

$$
v(\xi) \geq v\left(\xi_{0}\right)+\left\langle q, \overline{\xi_{0}^{-1} \cdot \xi}\right\rangle+o\left(\left|\xi_{0}^{-1} \cdot \xi\right|_{G}\right) .
$$

Jets can also be characterized by test functions $\psi$ as follows, see [16] in the Heisenberg Group case.

Let $u$ defined on a neighborhood of a point $\xi_{0}$. Suppose that $\psi$ is a $C^{1}$ function touching $u$ from above at $\xi_{0}$,

$$
u\left(\xi_{0}\right)=\psi\left(\xi_{0}\right)
$$

and

in a neighborhood of $\xi_{0}$. Then the vector

$$
u(\xi) \leq \psi(\xi)
$$

$$
\left(D_{\mathrm{h}} \psi\left(\xi_{0}\right)\right) \in J_{u}^{1,+}\left(\xi_{0}\right) .
$$

Moreover, every vector

is of the form

$$
\eta \in J_{u}^{1,+}\left(\xi_{0}\right)
$$

$$
\left(D_{\mathrm{h}} \psi\left(\xi_{0}\right)\right)
$$

for some $C^{1}$ function $\psi$ that touches $u$ from above at $\xi_{0}$.

A similar statement holds for $J_{u}^{1,-}\left(\xi_{0}\right)$ replacing "touching from above" by "touching from below".

An upper semicontinuous function $u$ is a viscosity subsolution of a first order equation of the form

$$
F\left(\xi, u, D_{h} u\right)=0
$$

where $F$ is continuous in all variables and increasing in $u$ if, for any $\xi_{0}$ and every $p \in J_{u}^{1,+}\left(\xi_{0}\right)$, the inequality holds:

$$
F\left(\xi_{0}, u\left(\xi_{0}\right), p\right) \leq 0 .
$$

A lower semicontinuous function $v$ is a viscosity supersolution of (3.3) if for any $\xi_{0}$ and every $q \in J_{v}^{1,-}\left(\xi_{0}\right)$, then:

$$
F\left(\xi_{0}, v\left(\xi_{0}\right), q\right) \geq 0 .
$$

A continuous function $u$ that is both a viscosity subsolution and a viscosity supersolution is called a viscosity solution. Uniform limits of viscosity solutions are viscosity solutions.

Let us start from a simple example of first order equation: the eikonal equation. The smooth gauge $|\cdot|_{G}$ is a viscosity subsolution of $\left|D_{h} u\right|=1$ in $B_{R}(\xi)-\{\xi\}$, with the boundary conditions $u=R$ on $\partial B_{R}$ and $u(\xi)=0$, see $[12,13]$. Let us consider a more general eikonal equation: $\left|D_{h} u\right|=C$ and prove that every subsolution is Lipschitz continuous with respect to the smooth gauge. By Pansu Theorem, it is also almost everywhere differentiable. The Lie bracket generating property transports the Lipschitz continuity from the horizontal layer to the entire group. In fact, control theory guarantees the continuity only for horizontally connected points. For arbitrary points, the Lie geometry plays the major role.

Theorem 3. Any bounded viscosity solution of $\left|D_{h} u\right| \leq C$ satisfies:

$$
|u(\xi \cdot \eta)-u(\xi)| \leq C|\eta| .
$$


Proof. First step: Without loss of generality we can assume that $\xi$ is equal to the origin $\underline{0}$. We shall prove the inequality for any horizontal $\eta \in G_{1}$. This is done following the same proof of [6], using Theorem 5.21 of [3]. We write the norm of the horizontal gradient:

$$
\left|D_{h} u\right|=|\sigma(\xi) \nabla u|=\sup _{\eta \in G_{1},|\eta| \leq 1} \sigma^{T} \eta \cdot \nabla u
$$

where $\sigma$ is the $m \times N$ matrix with entries $a_{i j}, X_{i}=\sum_{j} a_{i j} \frac{\partial}{\partial x_{j}}$. We are considering the evolution of a horizontal curve starting from the origin:

$$
\left\{\begin{array}{lll}
\gamma^{\prime}(t) & = & \sum_{i=1}^{m} \eta_{i} X_{i}(\gamma(t)) \\
\gamma(0) & = & \underline{0}
\end{array}\right.
$$

where $\eta \in \mathbb{R}^{m}$. The solution $y(s, \eta)$ can be parametrized using the dilations: $y(s, \eta)=\delta_{s}(\eta)$. Geometrically, it represents a horizontal curve joining the origin and $\eta \in G_{1}$.

The function $u$ is a viscosity subsolution of the Hamilton-Jacobi-Bellmann equation:

$$
\sup _{\eta \in G_{1},|\eta| \leq 1}\left\{\left\langle\eta, D_{h} u\right\rangle-C\right\}=0 .
$$

Hence $u$ satisfies:

$$
u(y(s, \eta))-u(y(t, \eta)) \leq(t-s)
$$

for $s \leq t$. Changing $\eta$ in $-\eta$ we get the assertion for horizontally connected points. The general case is achieved using the Lie bracket generating property. We write the point $\eta$ as direct sum of projection onto $G_{1}, G_{2}, \cdots, G_{r}$, namely $\eta=h_{1} \oplus h_{2} \oplus \cdots \oplus h_{r}$. We write the difference in a telescopic sum:

$$
\begin{gathered}
u(\underline{0})-u(\eta)=u(\underline{0})-u\left(h_{1}, 0, \cdots, 0\right)+u\left(h_{1}, 0, \cdots, 0\right)-u\left(h_{1}, h_{2}, \cdots, 0\right) \\
+\cdots+u\left(h_{1}, h_{2}, \cdots, h_{r-1}, 0\right)-u\left(h_{1}, h_{2}, \cdots, h_{r}\right) .
\end{gathered}
$$

The first difference is controlled by step 1 . To estimate the second one, we take $a=\left|h_{2}\right|^{\frac{1}{2}}$ and apply $C_{2}$ to the point $\left(h_{1}, 0, \cdots, 0\right)$. Iterating the construction, after a finite number of steps, we get the all group.

The telescoping sum is controlled by:

$$
\left|h_{1}\right|+\left|h_{2}\right|^{\frac{1}{2}}+\cdots+\left|h_{r}\right|^{\frac{1}{r}}
$$

Therefore, we reach the assertion, having a control in terms of the smooth gauge.

From now on, we will always consider Lipschitz continuity with respect to the Carnot smooth gauge.

Our Hamilton-Jacobi equations is of the form:

$$
\gamma u+H\left(\xi, D_{h} u\right)=0
$$

with $\gamma>0$. The Hamiltonian $H$ verifies:

$$
\begin{gathered}
\left|H\left(\xi^{\prime}, p\right)-H(\xi, p)\right| \leq m\left(\left|\xi^{-1} \cdot \xi^{\prime}\right|_{G}(1+|p|)\right) Q\left(\xi, \xi^{\prime}, p\right) \\
|H(\xi, 0)| \leq C_{3} \\
\lim _{|p| \rightarrow \infty} H(\xi, p)=+\infty \quad \text { uniformly in } \xi
\end{gathered}
$$

where $m(t) \rightarrow 0$ when $t \rightarrow 0$ and $Q\left(\xi, \xi^{\prime}, p\right)=\max \left\{\Phi(H(\xi, p)), \Phi\left(H\left(\xi^{\prime}, p\right)\right)\right\}$ and $\Phi$ is a continuous function from $\mathbb{R}$ into $\mathbb{R}^{+}$. Examples of Hamiltonians that satisfy our hypotheses are: $H(x, p)=\Psi\left(H_{o}(x, p)\right)$ where $H_{o}$ is a continuous function on $\mathbb{R}^{2 N}$ satisfying: $p \rightarrow H_{o}(x, p)$ convex, $H_{o}(x, \lambda p)=\lambda H_{o}(x, p), H_{o}(x, p) \geq 0$, 
$\left|H_{o}(x, p)-H_{o}(y, p)\right| \leq \omega\left(|x-y|(1+|p|)\right.$, with $\omega$ modulus of continuity such that $\lim _{s \rightarrow 0^{+}} \omega(s)=0$, for all $\lambda>0, x, y \in \mathbb{R}^{N}$. We assume also that $H_{o}$ is degenerate coercive in the sense that for some $\varepsilon>0$ $\sigma(x)\left([-\varepsilon, \varepsilon]^{m}\right) \subset \partial H_{o}(x, 0)$. Here $\partial H_{o}$ is the subdifferential of the convex function $H_{o}$ and $\sigma(x)$ is the $m \times N$ matrix as in the introduction. The function $\Psi$ is convex, increasing, $\Phi(0)=0$. The function $H_{o}$ plays the role of a generalized gauge in $G$, for example, one can take $H_{o}(\xi)=|\xi|_{C C}$.

Remark 1. The hypotheses on the Hamiltonian $H$ ensure that $-\gamma C_{3}$ and $\gamma C_{3}$ are respectively subsolution and supersolution of the equation (3.5). In particular, they are also Euclidean viscosity subsolution and supersolution. Perron's method for viscosity solutions [11] proves the existence of an Euclidean viscosity solution, that is also subelliptic viscosity solution. Also, a solution, as a consequence of the coercivity of $H$, verifies an equation of the form $\left|D_{h} u\right| \leq M$, for some $M>0$, so it is (subelliptic) Lipschitz continuous, see Theorem 3 .

It will remain to prove the uniqueness theorem. The proof for second order subelliptic equations has been done in [19]. The author uses the Euclidean theorem to get the jets and twist them into the subelliptic setting. For first order equations, we can proceed directly.

Theorem 4. Let $u$ a bounded subsolution and $v$ a bounded supersolution of equation (3.5). Then we have $u \leq v$ on $G$. As a consequence, the solution is unique.

Proof. Suppose, by contradiction, that $\sup (u-v)=\delta>0$. We define the functions:

$$
A(\xi, \eta)=\left|\xi^{-1} \cdot \eta\right|_{G}^{r} \quad \rho(\xi)=|\xi|_{G}^{r} .
$$

We have the following inequalities:

$$
\left|D_{h, \xi} A(\xi, \eta)\right| \leq C r A^{\frac{r-1}{r}}(\xi, \eta),\left|D_{h, \eta} A(\xi, \eta)\right| \leq C r A^{\frac{r-1}{r}}(\xi, \eta) .
$$

Consider for $\tau$ and $\alpha$ positive:

$$
\psi(\xi, \eta)=u(\eta)-v(\xi)-\tau A(\xi, \eta)-\alpha \rho(\xi)
$$

For every $\tau$ and $\alpha$, since $u$ and $v$ are bounded there is a maximum of $\psi$ attained in a point $\left(\xi_{\tau, \alpha}, \eta_{\tau, \alpha}\right)$. In particular, the point $\xi_{\tau, \alpha}$ is a minimum for $\xi \rightarrow v(\xi)+\tau A\left(\xi, \eta_{\tau, \alpha}\right)+\alpha \rho(\xi)$ and $v$ is a supersolution, we get that $p_{\tau, \alpha}=-\tau D_{h, \xi_{\tau, \alpha}} A\left(\xi_{\tau, \alpha}, \eta_{\tau, \alpha}\right)-\alpha D_{h} \rho\left(\xi_{\tau, \alpha}\right) \in J_{v}^{1,-}\left(\xi_{\tau, \alpha}\right)$ and plugging it into the equation:

$$
\gamma v\left(\xi_{\tau, \alpha}\right)+F\left(\xi_{\tau, \alpha}, p_{\tau, \alpha}\right) \geq 0
$$

From the other hand, the point $\eta_{\tau, \alpha}$ is a maximum for the function: $\eta \rightarrow u(\eta)-\tau A\left(\xi_{\tau, \alpha}, \eta\right)$ and $u$ is a viscosity subsolution:

$$
\gamma u\left(\eta_{\tau, \alpha}\right)+F\left(\eta_{\tau, \alpha}, q_{\tau, \alpha}\right) \leq 0
$$

where $q_{\tau, \alpha}=\tau D_{h, \eta_{\tau, \alpha}} A\left(\xi_{\tau, \alpha}, \eta_{\tau, \alpha}\right) \in J_{u}^{1,+}\left(\eta_{\tau, \alpha}\right)$.

Now the coercitivity condition on $H$ and the viscosity subsolution property of $u$ guarantee that $u$ is in fact a viscosity subsolution of an eikonal equation $\left|D_{h} u\right| \leq M$ for some $M>0$. We then apply the Lipschitz continuity to get:

which implies

$$
\tau A\left(\xi_{\tau, \alpha}, \eta_{\tau, \alpha}\right) \leq u\left(\eta_{\tau, \alpha}\right)-u\left(\xi_{\tau, \alpha}\right) \leq M\left|\xi_{\tau, \alpha}^{-1} \cdot \eta_{\tau, \alpha}\right|_{G} \leq M A\left(\xi_{\tau, \alpha}, \eta_{\tau, \alpha}\right)^{\frac{1}{r}}
$$

$$
A\left(\xi_{\tau, \alpha}, \eta_{\tau, \alpha}\right) \leq\left(\frac{M}{\tau}\right)^{\frac{r}{r-1}}
$$

From the other hand, writing $\psi\left(\xi_{\tau, \alpha}, \eta_{\tau, \alpha}\right) \geq \psi\left(\xi_{\tau, \alpha}, \xi_{\tau, \alpha}\right)$, we get:

$$
\tau A\left(\xi_{\tau, \alpha}, \eta_{\tau, \alpha}\right)+\alpha \rho\left(\xi_{\tau, \alpha}\right) \leq C .
$$


We get

$$
\begin{array}{cc}
\left|\xi_{\tau, \alpha}^{-1} \eta_{\tau, \alpha}\right|_{G} \rightarrow 0 & (\tau, \alpha) \rightarrow(+\infty, 0) \\
\alpha \rho\left(\xi_{\tau, \alpha}\right) \rightarrow 0 & (\tau, \alpha) \rightarrow(+\infty, 0) \\
M_{\tau, \alpha} \rightarrow M & (\tau, \alpha) \rightarrow(+\infty, 0)
\end{array}
$$

Taking into account the hypothese on $H$ :

$$
\begin{gathered}
\gamma\left(u\left(\eta_{\tau, \alpha}\right)-v\left(\xi_{\tau, \alpha}\right)\right) \leq H\left(\eta_{\tau, \alpha}, q_{\tau, \alpha}\right)-H\left(\xi_{\tau, \alpha}, p_{\tau, \alpha}\right) \\
H\left(\eta_{\tau, \alpha}, q_{\tau, \alpha}\right)-H\left(\xi_{\tau, \alpha}, q_{\tau, \alpha}\right)+H\left(\xi_{\tau, \alpha}, q_{\tau, \alpha}\right)-H\left(\xi_{\tau, \alpha}, p_{\tau, \alpha}\right) \\
\leq m\left(\left|\xi_{\tau, \alpha}^{-1} \eta_{\tau, \alpha}\right|_{G}\left(1+\left|q_{\tau, \alpha}\right|\right)\right)+n_{\tau}\left(\alpha\left|\xi_{\tau, \alpha}\right|\right)
\end{gathered}
$$

where $n(\cdot)$ is a modulus of continuity of $H$ on the set $G \times \bar{B}_{s_{\tau}}$ and $s_{\tau}=\max \{\|u\|,\|v\|, 1+\tau\}$. To conclude, we fix $\tau$ and let $\alpha$ goes to zero, then we let $\tau$ goes to $+\infty$.

\section{Homogenization problems}

Consider the cube $Q=\left[-\frac{1}{2}, \frac{1}{2}\right)^{N}$ and construct a tiling of $G$ moving only from horizontal points of the form $(\underline{k}, 0), \underline{k} \in \mathbb{Z}^{m}$, where $(\underline{k}, 0) \in G_{1}, Q_{k}=(\underline{k}, 0) \cdot Q$. The bracket generating property yields the following property:

Lemma 1. For every point $\xi \in G$ there exists a point $\xi_{0} \in Q$ and a finite number of group actions generated by elements of the form $(\underline{k}, 0), \underline{k} \in \mathbb{Z}^{m}$ that applied to $\xi_{0}$ give $\xi$.

Proof. We put $k_{i}=\left[x_{i}+\frac{1}{2}\right]$, for $i=1 \cdots m$, where [.] stand for the integer part; hence $\tilde{x}_{i}=x_{i}-k_{i} \in\left[-\frac{1}{2}, \frac{1}{2}\right)^{m}$. We consider $\bar{k}=\left(k_{1}, \cdots, k_{m}\right) \in \mathbb{Z}^{m}$ and compute

$$
(\bar{k}, 0, \cdots, 0) \cdot \tilde{\xi}
$$

The group operation gives a point with the first $m$ coordinates equal to the first $m$ coordinates of $\xi$ and the $m+1$ equal to $\tilde{\xi}_{m+1}+Q_{2}$, where $Q_{2}$ is an homogeneous polynomial of degree 2 in the horizontal variables. Using the Lie bracket generating property towards the mappings $C_{\ell}$ we could solve a system of equations in terms of the components of $\xi_{0}$.

We will adopt this latter one as an intrinsic pavage and therefore the corresponding notion of periodicity, we will call it $G$-periodicity.

Definition 2. A function $f$ defined on a Carnot Group $G$ is said $G$-periodic iff

$$
f((\underline{k}, 0) \cdot \xi)=f(\xi) \quad \forall \underline{k} \in \mathbb{Z}^{m}, \forall \xi \in Q
$$

Consider now the action of the dilatations of the group:

$$
Q_{k}^{\varepsilon}=\delta_{\varepsilon}\left(Q_{k}\right)=\delta_{\varepsilon}((\underline{k}, 0) \cdot Q) \text {. }
$$

We notice that $\left\{Q_{k}^{\varepsilon}\right\}$ is still a tiling of $G$. Furthermore, if $f$ is $G$-periodic, then $f\left(\delta_{\frac{1}{\varepsilon}} \xi\right)$ is $\varepsilon G$-periodic.

In this context, homogenizations problems are:

$$
u^{\varepsilon}+H\left(\xi, \delta_{\frac{1}{\varepsilon}} \xi, D_{h} u^{\varepsilon}(\xi)\right)=0
$$


with $\eta \rightarrow H(\xi, \eta, p) G$-periodic for each $(\xi, p) \in G \times \mathbb{R}^{m}$. The function $H$ is defined on $\mathbb{R}^{2 N} \times \mathbb{R}^{m}$ and satisfies for all $\xi, \xi^{\prime}, \eta, \eta^{\prime} \in \mathbb{R}^{N}$ and all $p, p^{\prime} \in \mathbb{R}^{m}$ :

$$
\begin{gathered}
\left|H\left(\xi^{\prime}, \eta^{\prime}, p^{\prime}\right)-H(\xi, \eta, p)\right| \leq C\left(\left|\xi^{-1} \cdot \xi^{\prime}\right|_{G}+\left|\eta^{-1} \cdot \eta^{\prime}\right|_{G}+\left|p-p^{\prime}\right|\right) \\
\nu|p|-C_{3} \leq H(\xi, \eta, p) \leq \nu|p|+C_{3} .
\end{gathered}
$$

Remark 2. From the previous section we do know that it exists a sequence of solutions of the (4.1); moreover this sequence is equibounded and equilipschitz. In fact, $-C_{3}$ and $C_{3}$ are, respectively, subsolution and supersolution of equations (4.1) and, therefore, by comparison $\left\|u_{\varepsilon}\right\|_{\infty} \leq C$. In addition, using the coercivity of $H$, we get that $\left|D_{h} u_{\varepsilon}\right| \leq C$ in the viscosity sense, that implies, by Theorem 3 , the equilipschitz bound. So, up to a subsequence, it converges uniformly, as $\varepsilon$ tends to zero, to a continuous function $u$, the solution of the homogenized problem, see Theorem 6 .

The nonlinear eigenvalue problem for the effective Hamiltonian is:

$$
H\left(\xi, \eta, p+D_{h} v(\eta)\right)=\lambda
$$

and then the effective Hamiltonian $\bar{H}$ is

$$
\bar{H}(\xi, p)=\lambda
$$

for every $(\xi, p) \in G \times \mathbb{R}^{m}$. The uniqueness of $\lambda$ depending on $(\xi, p)$ is a consequence of the comparison theorem. As in the Euclidean case, (see [14],[7]), one consider a sequence of approximated problems:

$$
\alpha v_{\alpha}(\eta)+H\left(\xi, \eta, p+D_{h} v_{\alpha}(\eta)\right)=0
$$

and study the asymptotic behaviour as $\alpha$ tends to zero. We already know that there exists a solution $v_{\alpha}$ bounded and Lipschitz continuous,that is also periodic. It can be viewed indeed (see [14]) that the limit of $\alpha v_{\alpha}(\eta)$ does not depend on $\eta$ and that a solution $(\lambda, v)$ of $(4.2)$ is given by

$$
\lambda=-\lim _{\alpha \rightarrow 0} \alpha v_{\alpha} \quad v(\eta)=\lim _{\alpha \rightarrow 0}\left(v_{\alpha}-i n f_{Q} v_{\alpha}\right) .
$$

Theorem 5. For each $p$ there exists a unique $\lambda$ such that there exists $v$ viscosity solution of (4.2).

Proof. From Theorem 4 we know that there exists unique $v_{\alpha}$ that is also Lipschitz and, from the uniqueness, it follows that is periodic. Moreover

$$
-\sup _{\eta} H(\xi, \eta, p) \leq-\alpha v_{\alpha}(\eta) \leq-\inf _{\eta} H(\xi, \eta, p)
$$

and from the coercivity of $H$, we also get:

$$
\left\|D_{h} v_{\alpha}\right\|_{\infty} \leq C
$$

for some $C$ independent of $\alpha$. Set $\tilde{v_{\alpha}}=v_{\alpha}-i n f_{Q} v_{\alpha}$ and observe that $\tilde{v_{\alpha}}$ is periodic and equilipschitz. Up to a subsequence, we may assume that $\left(-\alpha v_{\alpha}, \tilde{v_{\alpha}}\right)$ converges uniformly to some $(\lambda, v)$. From the stability property of viscosity solutions, $v$ is a solution of (4.2). Suppose now that there exists another couple $(\mu, w)$, solution of the cell problem, with $\lambda \neq \mu$. We may assume that $\lambda<\mu$ is the case. Since if $w$ is a solution of

$$
H\left(\xi, \eta, p+D_{h} w(\eta)\right)=\mu
$$

then so is $w+c$; therefore, we can also assume that $w>v$. Choose $\alpha$ so that $\lambda+\alpha v \leq \mu+\alpha w$. We notice that $v, w$ are respectively the unique viscosity solutions of

$$
\begin{gathered}
H\left(\xi, \eta, p+D_{h} v(\eta)\right)+\alpha v(\eta)=\lambda+\alpha v(\eta) \\
H\left(\xi, \eta, p+D_{h} w(\eta)\right)+\alpha w(\eta)=\mu+\alpha w(\eta) .
\end{gathered}
$$

Then, in particular, $w$ is a super solution of (4.4), and by comparison, $v \leq w$, that is a contradiction. 
We now conclude by proving the uniform convergence of $u^{\varepsilon}$ towards the solution of the homogenized problem, using the perturbed test functions method adapted to Hamilton-Jacobi equations.

Theorem 6. The sequence $u^{\varepsilon}$ converges uniformly to the solution of the homogenized equation:

$$
u+\bar{H}\left(\xi, D_{h} u\right)=0
$$

Proof. We consider a point $\xi_{0}$ and a test function $\phi$ such that $u-\phi$ has a strict local maximum at $\xi_{0}$, we need to prove that

$$
\phi\left(\xi_{0}\right)+\bar{H}\left(\xi_{0}, D_{h} \phi\left(\xi_{0}\right)\right) \leq 0 .
$$

We start by solving the cell problem (4.2) at the point $\xi_{0}$ and with $p=D_{h} \phi\left(\xi_{0}\right)$

$$
H\left(\xi_{0}, y, D_{h} \phi\left(\xi_{0}\right)+D_{h} \psi(y)\right)=\bar{H}\left(\xi_{0}, D_{h} \phi\left(\xi_{0}\right)\right)
$$

The function $\psi$ is $G$-periodic and defined up to a constant, we normalize it, by requiring that $\int_{Q} \psi=1$. Since $\psi$ is a.e. differentiable, we take a test function $\chi$ touching $\psi$ from above at the point $\delta_{\frac{1}{\varepsilon}}\left(\xi_{0}\right)$. Consider the perturbed test function:

$$
\phi^{\varepsilon}(\xi)=\phi(\xi)+\varepsilon \chi\left(\delta_{\frac{1}{\varepsilon}}(\xi)\right)
$$

Since $u-\phi$ has a strict local maximum at $\xi_{0}$ and, up to a subsequence, $u^{\varepsilon} \rightarrow u, \phi^{\varepsilon} \rightarrow \phi$ uniformly, we see that for each $\varepsilon, u^{\varepsilon}-\phi^{\varepsilon}$ has a local maximum at some point $\xi_{\varepsilon}$, with $\xi_{\varepsilon} \rightarrow \xi_{0}$ as $\varepsilon \rightarrow 0$. By using that $u^{\varepsilon}$ is a solution of (4.1), we get:

$$
\phi^{\varepsilon}\left(\xi_{\varepsilon}\right)+H\left(\xi_{\varepsilon}, \delta_{\frac{1}{\varepsilon}}\left(\xi_{\varepsilon}\right), D_{h} \phi^{\varepsilon}\left(\xi_{\varepsilon}\right)\right) \leq 0
$$

We compute:

$$
D_{h} \phi^{\varepsilon}\left(\xi_{\varepsilon}\right)=D_{h} \phi\left(\xi_{\varepsilon}\right)+D_{h} \chi\left(\delta_{\frac{1}{\varepsilon}}\left(\xi_{\varepsilon}\right)\right)=D_{h} \phi\left(\xi_{0}\right)+D_{h} \chi\left(\delta_{\frac{1}{\varepsilon}}\left(\xi_{\varepsilon}\right)\right)+o(1)
$$

since the horizontal gradient commutes with dilations and $\xi_{\varepsilon}$ converges to $\xi_{0}$.

By inserting $D_{h} \phi^{\varepsilon}\left(\xi_{\varepsilon}\right)$ in (4.8) and use the uniform convergence of $\phi^{\varepsilon}$ to $\phi$ and the continuity of $H$ :

$$
\phi\left(\xi_{0}\right)+H\left(\xi_{0}, \delta_{\frac{1}{\varepsilon}}\left(\xi_{\varepsilon}\right), D_{h} \phi\left(\xi_{0}\right)+D_{h} \chi\left(\delta_{\frac{1}{\varepsilon}}\left(\xi_{\varepsilon}\right)\right) \leq o(1) .\right.
$$

We now set $y=\delta_{\frac{1}{\varepsilon}}\left(\xi_{\varepsilon}\right)$ in (4.7) and substitute with $\bar{H}$, to conclude as $\varepsilon \rightarrow 0$. Analogously, starting from a strict local minimum for $u-\phi$, we can show the opposite inequality.

\section{APPENDIX}

We have defined the function:

$$
\begin{aligned}
A(\xi, \eta)=\left\{\left[\sum_{j=1}^{m}\left(x_{1, j}-y_{1, j}\right)^{2}\right]^{r !}\right. & +\left[\left(x_{m+1}-y_{m+1}+Q_{2}\right)^{2}\right]^{\frac{r !}{2}} \\
& \left.+\cdots+\left[\left(x_{m+r-1}-y_{m+r-1}+Q_{r-1}\right)^{2}\right]^{\frac{r !}{r-1}}+\left[\left(x_{m+r}-y_{m+r}+Q_{r}\right)^{2}\right]^{(r-1) !}\right\}^{\frac{r}{2 r !}} \\
& +
\end{aligned}
$$

Let us develop the calculations for:

$$
\left|D_{h, \xi} A(\xi, \eta)\right| \leq C r A^{\frac{r-1}{r}}(\xi, \eta) .
$$


Denote by $B$ the argument of $A$ inside the graph parenthesis:

$$
\begin{gathered}
B=\left[\sum_{j=1}^{m}\left(x_{1, j}-y_{1, j}\right)^{2}\right]^{r !}+\cdots+\left[\left(x_{m+r-1}-y_{m+r-1}+Q_{r-1}\right)^{2}\right]^{\frac{r !}{r-1}}+\left[\left(x_{m+r}-y_{m+r}+Q_{r}\right)^{2}\right]^{(r-1) !} \\
=I_{1}^{r !}+I_{2}^{\frac{r !}{2}}+\cdots+I_{r-1}^{\frac{r !}{r-1}}+I_{r}^{(r-1) !} \\
X_{j, \xi} A(\xi, \eta)=X_{j, \xi}\{B(\xi, \eta)\}^{\frac{r}{2 r !}} \\
=\frac{r}{2 r !}\{B\}^{\frac{r}{2 r !}-1} X_{j, \xi} B .
\end{gathered}
$$

Observe that the exponent $\frac{r}{2 r !}-1$ is negative. Recall now the formula (2.1) in a simplified version accordingly to our notations:

$$
X_{j}(g)=\frac{\partial}{\partial x_{j}}+\sum_{s=m+1}^{m+r} b_{s, j}\left(x_{1}(g), \cdots, x_{s-1}(g)\right) \frac{\partial}{\partial x_{s}}, \quad j=1, \cdots, m
$$

where each $b_{s, j}$ is a homogeneous polynomial of weighted degree $s-m$ and observe that $Q_{j}$ are homogeneous polynomial of weighted degree $j$. We get:

$$
\begin{aligned}
{\left[C_{1} I_{1}^{r !-1} I_{1}^{\frac{1}{2}}+\right.} & \left.\cdots+C_{r-1} I_{r-1}^{\frac{r !}{r-1}+\frac{1}{2}} R_{r-2}\right]+C_{r} I_{r}^{(r-1) !+\frac{1}{2}} R_{r-1} \\
& +b_{m+1, j}\left\{C_{2} I_{1}^{\frac{r !}{2}-1} I_{2}^{\frac{1}{2}}+\cdots+C_{r-1} I_{r-1}^{\frac{r !}{r-1}+\frac{1}{2}} R_{r-3}+C_{r} I_{r}^{(r-1) !+\frac{1}{2}} R_{r-2}\right\}+\cdots+b_{m+r, j} C_{r} I_{r}^{(r-1) !+\frac{1}{2}}
\end{aligned}
$$

where $R_{t}$ are homogeneous polynomial of weighted degree $t$. To conclude, observe that the polynomial $R_{j}$ are equivalent to the polynomial $Q_{j}$ that appear in the group operation.

Acknowledgements. The author was supported in part by Prin 2005 "Calcolo delle Variazioni" and by G.N.A.M.P.A. Project 2005: "Partial Differential Equations and Control Theory".

\section{REFERENCES}

[1] O. Alvarez, and M. Bardi, Viscosity solutions methods for singular perturbations in deterministic and stochastic control. SIAM J. Control Optim. 40 (2001) 1159-1188.

[2] M. Arisawa, Quasi-periodic homogenizations for second-order Hamilton-Jacobi-Bellmann equations. Adv. Sci. Appl. 11 (2001) 465-480.

[3] M. Bardi and I. Capuzzo Dolcetta, Optimal control and viscosity solutions of Hamilton-Jacobi-Bellman equations. Birkhäuser Boston, Boston, MA (1997).

[4] G. Barles, Solutions de viscosité des équations de Hamilton-Jacobi. Springer-Verlag, Math. Appl. 17 (1994).

[5] A. Bellaïche and J.-J. Risler, ed., Sub-Riemannian Geometry. Birkhäuser, Progress. Math. 144 (1996).

[6] I. Birindelli and J. Wigniolle, Homogenization of Hamilton-Jacobi equations in the Heisenberg Group. Commun. Pure Appl. Anal. 2 (2003) 461-479.

[7] I. Capuzzo Dolcetta and H. Ishii, On the rate of convergence in homogenization of Hamilton-Jacobi equations. Indiana University Math. J. 50 (2001) 1113-1129.

[8] L.C. Evans, The perturbed test function method for viscosity solutions of nonlinear PDE. Proc. Roy. Soc. Edinburgh 11A (1989) 359-375.

[9] L.C. Evans, Periodic homogenization of certain fully nonlinear PDE. Proc. Roy. Soc. Edinburgh 120 (1992) $245-265$.

[10] G.B. Folland and E.M. Stein, Hardy spaces on homogeneous groups. Princeton University Press, Princeton, N.J., University of Tokyo Press, Tokyo. Math. Notes 28 (1982)

[11] H. Ishii, Perron's method for Hamilton-Jacobi equations. Duke Math. J. 55 (1987) 369-384.

[12] P. Juutinen, G. Lu, J. Manfredi and B. Stroffolini, Convex functions on Carnot Groups, to appear in Revista Mathematica Iberoamericana. 
[13] G. Lu, J. Manfredi and B. Stroffolini, Convex functions on the Heisenberg Group. Calc. Var. Partial Differential Equations 19 (2004) 1-22.

[14] P.L. Lions, G. Papanicolau and R.S. Varadhan, Homogenization of Hamilton-Jacobi equations, preprint (1986).

[15] J. Manfredi, Nonlinear subelliptic equations on Carnot Groups. Notes of a course at the School on Analysis and Geometry, Trento (2003).

[16] J. Manfredi and B. Stroffolini A Version of the Hopf-Lax Formula in the Heisenberg Group. Comm. in Partial Differential Equations 27 (2002) 1139-1159.

[17] R. Montgomery, A Tour of Subriemannian Geometries, their geodesics and applications. American Mathematical Society, Providence, RI. Math. Surveys Monographs 91(2002).

[18] R. Monti and F. Serra Cassano, Surface measures in Carnot-Carathéodory spaces. Calc. Var. 13 (2001) 339-376.

[19] D. Morbidelli, Fractional Sobolev norms and structure of Carnot-Caratheodory balls for Hörmander vector fields. Studia Math. 139 (2000) 213-244.

[20] A. Nagel, E.M. Stein and S. Wainger, Balls and metrics defined by vector fields I: basic properties. Acta Math. 137 (1976) $247-320$. 